\title{
Working Group on Massive Stars
}

\author{
CHAIRPERSON: Claus Leitherer \\ ORGANIZING COMMITTEE: Paul Crowther, Philippe Eenens, \\ Alex Fullerton, Gloria Koenigsberger, Phil Massey, George Meynet, \\ Tony Moffat, Stan Owocki, and Joachim Puls
}

\begin{abstract}
This report covers the activity period 2002-2005 of the IAU Working Group on Massive Stars
\end{abstract}

\section{Mission Statement}

Our group's focus is the study of massive stars, both individually and in resolved and unresolved populations. These objects populate the upper part of the HertzsprungRussell diagram. On the main-sequence these stars are spectroscopically identified as types $\mathrm{O}$ and early B, while later evolutionary stages encompass supergiants and WolfRayet stars. Our group also studies some stars of lower mass, which may show many features similar or related to those present in massive stars, and thus may improve our understanding of the physical processes occurring in massive stars.

\section{Organizational Matters}

The members of the Working Group elected Paul Crowther, Alex Fullerton, Gloria Koenigsberger, Claus Leitherer, Phil Massey, George Meynet, and Joachim Puls into the Organizing Committee (OC). The new OC members joined Tony Moffat, Stan Owocki, and Philippe Eenens who are still completing their terms in the 10-person OC. The OC then elected Claus Leitherer as Chairperson.

After thorough debate, the OC voted for a name change of the Working Group from "Hot Massive Stars" to "Massive Stars". This name change reflects the tight interrelation of research on hot and cool stars in the upper Hertzsprung-Russell diagram (see below).

The OC created and approved a set of by-laws. The by-laws are posted on the Working Group website at http://www. astroscu.unam.mx/massive__stars/.

The distribution list of our members and their e-mail addresses was updated. As of 2005, there are 429 members in the Working Group at research and educational institutions.

\section{Website Development}

A new web portal was designed and implemented on a server located at UNAM (http: //www . astroscu . unam.mx/massive__stars/). After an open call soliciting expressions of interest, the OC was pleased to select Raphael Hirschi (University of Basel) as the new webmaster. Back issues are posted on the Working Group website and provide an invaluable record of scientific developments in the field, particularly the emergence of new "hot topics".

Among other innovations, the new web portal offers a discussion group page and an automatic Newsletter submission interface. This new interface allows the members of 
the Working Group to submit their Newsletter abstracts and circulates newly received abstracts to registered members.

\section{Science Support}

The Massive Star Newsletter, edited by Philippe Eenens, continues to be the main means of communication and science dissemination in our Working Group. As of October 2005, 87 issues of the Newsletter have been published. Back issues are posted on the Working Group website.

The OC is currently discussing routes to foster more interactions between researchers working on hot and cool massive stars. Scientific areas that would benefit from such a dialog are the evolutionary relation between the hot and the cool part of the HRD, W$\mathrm{R} / \mathrm{LBV}$ progenitors, dust formation in RSG/AGB vs. W-R stars, wind hydrodynamics, or abundance determinations from hot vs. cool stars.

A second area of research with a strong need for interaction concerns rotating stars. Recent work by the Geneva group points to the likelihood that rotational spin-up plays an important role in the evolution of massive stars. Be stars may be a key laboratory for studying its nature and consequences.

The OC is concerned about a fair gender and ethnic representation in committees and initiatives of interest to our Working Group. The OC feels the best way to promote a fair representation is to encourage its members from minority groups to actively volunteer for service. This is particular relevant for junior members, who tend to be underrepresented. A major challenge for the WG is to improve its visibility with our junior members and with the community at large. Many junior massive-star researchers are not aware of the efforts of the Working Group, and they do not know the decision making process. The OC should leverage the enormous scientific potential of our members in order to advance our field of research.

\section{Conference Planning}

A proposal for a Joint Discussion during the XXVI ${ }^{\text {th }}$ General Assembly by several OC members was accepted by the IAU. The 1-day meeting Calibrating the Top of the Stellar Mass-Luminosity Relation is planned for August 16, 2006. The goal of this Joint Discussion is to bring together theorists and observers from the stellar and extra-galactic communities to discuss the properties of the most massive stars and the implications for cosmological studies. The meeting will focus on a set of themes that follow from fundamental stellar astronomy, such as mass determinations in binary stars, to recent modeling of atmospheres and evolution, to the significance of massive stars for the ecology of the host galaxy, and finally to a critical assessment of the properties of the first generation of stars in the universe. The meeting organizers are Norbert Langer, Claus Leitherer (Chair), Tony Moffat, Stan Owocki, and Joachim Puls. The website is http://www.stsci.edu/science/starburst/Prague/.

The OC supported a proposal for a planned IAU symposium entitled "Massive Stars as Cosmic Engines" to be held on Kauai (Hawaii, USA) between December 10-14, 2007.

The theme of the conference will be how massive stars shape the universe from the nearby universe to high red-shift galaxies. They form in starbursts, chemically enrich the interstellar medium, inject energy via their stellar winds and core-collapse supernovae, drive the interstellar medium out of galaxies, enriching the intergalactic medium. The major observational constraints at high redshift, Lyman-break galaxies and damped 
Lyman- $\alpha$ absorbers, are direct detection of massive stars via their UV continua and stellar winds and indirectly via the ionized ISM. Paul Crowther and Joachim Puls are the co-chairs of the SOC.

\section{Acknowledgements}

The OC gratefully acknowledges logistic support for several teleconferences by STScI. The Institute of Astronomy of UNAM provided a new disk drive in support of the web server. UNAM and STScI provided technical support through Liliana Hernandez and Julia Chen.

Claus Leitherer Chairperson of the Working Group 